\title{
Laboratory assessment of thermal transmittance of homogeneous building elements using infrared thermography
}

\author{
by I. Simões ${ }^{*}$ N. Simões ${ }^{* * *}$, A. Tadeu ${ }^{* * * *}$, J. Riachos ${ }^{* * *}$ \\ ${ }^{*}$ CICC, Department of Civil Engineering, University of Coimbra, Pólo II, Rua Luís Reis Santos, 3030-788 \\ Coimbra, Portugal, \{ mivsimoes, nasimoes, tadeu\}@itecons.uc.pt \\ **ITeCons - Instituto de Investigação e Desenvolvimento Tecnológico em Ciências da Construção, Rua Pedro \\ Hispano, 3030-289 Coimbra, Portugal \\ *** Department of Civil Engineering, University of Coimbra, Rua Luís Reis Santos - Pólo II, 3030-788 \\ COIMBRA, PORTUGAL, joana-riachos@hotmail.com.
}

\begin{abstract}
Due to a growing concern with the assessment of the thermal performance of building envelopes, in particular during energy audits and energy certification processes, it has become increasingly important to use inspection techniques to determine the thermal transmittance values (U-value) of building elements. One of the techniques that may be explored to evaluate the thermal behaviour of the envelope is Infrared thermography (IRT). As the accuracy of the thermal transmittance estimation is dependent on the state of the heat transfer, having steady-state or near steady-state conditions is crucial to get reliable measurements and a stable temperature difference between inside and outside is mandatory. With this in mind the authors intend to evaluate the application of IRT to U-value determination studies. For this reason, a set of laboratory measurements were performed. A steady temperature difference was imposed in 4 different homogeneous materials used to build a box that is heated using a thermal resistance. IRT temperatures were measured on the outside surfaces of the box, and are then used to estimate the U-value. Those results are compared with the U-value calculated using the thermal conductivity obtained by means of the guarded hot plate method.
\end{abstract}

\section{Introduction}

In the European Union (EU), nearly $40 \%$ of final energy consumption is attributable to housing. Tackling energy consumption in European buildings is vital and one of the measures that have been implemented has been the energy certifications of buildings ([1], [2]). The certification of existing buildings allows the identification of improvement measures. With that purpose, an adequate knowledge of the existing energy consumption profile of the building is important. To better understand the thermal performance of a building it is necessary to characterize its envelope, namely, by obtaining the thermal transmittance (U-value) of the building solutions ([3], [4]). However, determination of the $\mathrm{U}$-values is not easily performed on existing buildings, when the composition of the building elements is unknown. Destructive or sophisticated (non-invasive) approaches are needed. The first allows the $U$ estimation after the identification of the components, however a reparation is always necessary. Using non-invasive methods, we may notice that they are dependent of the climatic conditions and their limitations and uncertainty should not be neglected.

Different standards have indicated diverse methodologies to evaluate the thermal performance of building materials and construction solutions, e.g. ISO 6946 [5] and ISO 10211 [6], assuming steady-state conditions. ISO 6946 [5] assumes that building elements consist of thermally homogeneous layers with known thermal conductivity. It is only applicable to opaque building elements and it allows geometrical effects and air voids to be taken into account. More precise results can be obtained by using a numerical method in accordance with ISO 10211 [6].

As an alternative, U-values can be found by laboratory tests. For this purpose, a device composed of two chambers, one hot and the other cold, placed on either side of the specimen has been used for many years. This apparatus is today generally called a "hot-box". Standards such as EN ISO 8990 [7], widely used in Europe, the American ASTM C1363-05 [8] and the Russian Gost 26602.1-99 [9], describe the design requirements for this apparatus, the measurement technique and the test procedure followed in order to obtain the $U$-value. These three approaches are compared in Asdrubali and Baldinelli [10]. Their work is focused on the measurement procedures, calibration process and the uncertainties of each. The hot-box has also been used to validate analytical and numerical approaches ([11], [12]). Despite the U-value calculation being based on steady-state conditions, as defined in the above standards, the hot-box apparatus may be also used in transient conditions, as did Gao et al. [11], for estimating heat loss of hollow blocks wall and Burch et al. [13], for a multilayer masonry wall.

Currently, the available approaches to evaluate the U-value in existing buildings are: non-destructive methods (direct measurement of the heat flux ([14], [15]) using heat flux sensors or infrared thermography ([16], [17], [18]) or destructive methods (direct measure of layer thickness and determination of their conventional resistance). In the case of the destructive test, the methods proposed by the international standards ISO 6946 [5] and ISO 10211 [6] can be used to determine thermal transmittance. In this process, a guarded hot plate or a heat flow meter is recommended to find the thermal conductivity of the materials, proceeding as described in ISO 8301 [20] and ISO 8302 [21]. Giuseppe Desogus et 
al. [19] compared the results of thermal resistance (R-value) of a wall obtained using two methods: a non-destructive method (involving measuring the heat-flow rate through the test wall and its surface temperatures) and the destructive method. This comparison shows that the R-values measured by the non-destructive method (considering a temperature amplitude of $10^{\circ} \mathrm{C}$ between opposite wall surfaces) and the one calculated from the destructive method can have a variation of $16 \%$.

The non-destructive technique that uses heat flux sensors is described in the international standard ISO 9869 [22]. U-values can be found by measuring both the heat flow through the building element and the temperature on both sides of it. If the system is under steady-state conditions, the U-value can be very accurate. However, since exterior conditions are always changing, is not usual to find steady-state conditions during in-situ measurements. Two main approaches can then be applied, by recording the heat flow rate and temperatures over a long period in such a way that allows a good estimation of equivalent steady-state behavior or, by applying a dynamic model to take into account the surface temperature and heat flow rate variations. Using the first approach, results may be quite inaccurate unless the storage effect caused by thermal mass (inertia) is negligible for the heat flow rates in question. Changes in heat flow direction that occur during the day will may also lead to a decrease in the precision of measurements. The authors have published a numerical method to evaluate the R-value using a similar non-destructive technique (measuring heat fluxes, surface temperatures and wall thickness) for walls with a multilayer configuration subjected to a dynamic state [23]. The relative errors between the design values and the results obtained by the proposed method were shown to be below $8 \%$.

As mentioned before, another non-destructive technique that has been explored to evaluate the $U$-value is Infrared Thermography (IRT) ([16], [17], [18]). IRT is a non-contact technique using infrared radiation reader devices to acquire and analyse thermal pattern images. All objects radiate energy that is proportional to the surface emissivity of the material and to the fourth power of the absolute surface temperature, making it necessary to know with precision the emissivity of the measured surface. The IRT camera captures the radiation emitted by the object's surface, converting it into electrical signals and creating an image with the distribution of surface temperatures [24]. The hemispherical emissivity of a surface gives the ratio between the energy emitted by a surface of a particular material and the energy emitted by a perfect emitter (blackbody) at the same temperature and with an emissivity of one ([25], [26]). Emissivity values are expressed as a number between zero (perfect reflector, such as a mirror) and one (blackbody). A review of emissivity measurement techniques, and the importance of emissivity values to building diagnostics, was presented by Avdelidis and Moropoulou [26]. They used two approaches and found that the emissivity values of some materials are a function of their temperature; the first approach followed ASTM standard E1933-99 [27], while the other was an empirical procedure. They found that the emissivity values depend on the wavelength of the infrared thermographic region being analyzed.

As Balaras and Argiriou [25] state in the introduction of their work, temperature measurements using IRT are also dependent on other parameters such as ambient temperature, wind speed, distance to the target, air humidity and temperature reflected. The reflected temperature can be estimated according to ASTM 1862-97 [28]. This variable allows the correction of radiation emitted by the surface under analysis and by eliminating the influence of radiation emitted by the surrounding surfaces [29].

During in-situ inspections to the external side of the building envelope, other parameters may affect infrared measurements, such as rain and wind ([16], [30]). However, introducing the use of IRT technique in U-value determination has many advantages: it is a non-destructive and non-contact method; the measurements periods are short; it allows a preliminary evaluation of a wide area and the identification of the best place to perform final measurements (ensuring that measurements are performed in a relevant zone); it enables the distinction between different constructive solutions in a building (for example, it distinguishes a flat thermal bridge from the main constructive solution).

Albaciti and Tonelli [16] presented a method to calculate the U-value with IRT. To validate their method, they compared IRT results with measurements using a heat flux apparatus to obtain the wall heat fluxes and surface temperatures, and U-values were calculated using Fourier's law, taking into account radiation and convection phenomena. The two techniques were used in three in-situ case studies in Italy. The authors concluded that the IRT technique presented an error rate of $\pm 30 \%$, lower than the results obtained from heat flux sensor system.

Fokaides and Kalogirou [17] also applied the IRT technique to calculate U-values taking into account the influence of radiation and convection. The authors considered that the reflected temperature is numerically equal to the ambient temperature. For this reason, the authors used a temperature and relative humidity sensor to know the ambient temperature and relative humidity. The internal surface temperature was measured using and additional sensor. The authors reported errors from 10 to $20 \%$, when comparing U-values obtained with IRT and with results from a temperatures measuring instrument.

A similar procedure (IRT technique) was also presented by Vollmer and Möllmann [18], based on the study developed by Madding [31]. The thermal resistance (R-value given by the inverse of the $U$-value) obtained presented a percentage deviation of $5-12 \%$ from the theoretical value.

The three papers referred in the previous paragraphs ([16], [17] and [18]) proposed U-value calculation methods based on the radiation and convection phenomena estimation. In the equations proposed by Fokaides and Kalogirou [17] and by Vollmer and Möllmann [18] the radiation phenomenon takes into account the reflected temperature, while Albaciti and Tonelli [16] assumed that the reflected temperature was similar to the temperature of the environment. The equations provided by Fokaides and Kalogirou [17], differ from the ones given by Vollmer and Möllmann [18] because the latter considered the average values between surface and reflected temperatures. 
In the present paper, the application of IRT to estimate the thermal resistance of homogeneous materials is evaluated. With this purpose, the methods presented in [16], [17] and [18] were considered. An IRT camera was used to measure values of surface temperature, emissivity and reflected temperature. A set of laboratory measurements was performed to estimate the thermal resistance of 4 materials with different thermal conductivities (Medium-Density Fiberboard - MDF; Extruded Polystyrene - XPS; Natural Cork - NC; Cement Bonded Particle Board - CBPB). These materials were previously characterized by the Guarded Hot Plate (ISO 8302:1991 [32]) method, with the Mebtechnik Lambda GmbH, Dresden, Lambda-meter EP- 500 model, following the procedure described in EN 12667:2001 [33]. The materials were used to build a box and a temperature gradient between inside and outside the box (laboratory room) was created. The tests were carried out while maintaining a laboratory temperature of $(23 \pm 2){ }^{\circ} \mathrm{C}$ and a relative humidity of $(50 \pm 5)^{\circ} \mathrm{C}$.

To apply the different methods, seven variables were measured: emissivity, reflected temperature, ambient temperature, relative humidity, wind speed, inner and outer superficial temperatures. The emissivity and reflected temperature were determined according to the procedures described in ASTM Standard E1933-99 [27] and the ASTM Standard E1862-97 [28] respectively. The superficial temperature of the outer surfaces of the box was measured with an infrared thermal camera, after steady-state had been reached.

Both U-values, obtained by the proposed methodologies and determined theoretically (using the thermal conductivity determined by experimental tests) were compared.

\section{Methodology}

The nomenclature used in the methodology is presented in table 1.

Table 1. Nomenclature

\begin{tabular}{|c|c|c|}
\hline$A$ & Surface area & $\mathrm{m}^{2}$ \\
\hline E & Relative error & $\%$ \\
\hline$E_{\max }$ & Maximum relative error & $\%$ \\
\hline$e_{j}$ & Thickness of layer $j$ & $\mathrm{~m}$ \\
\hline$h_{c}$ & Convective coefficient & $\mathrm{W} /\left(\mathrm{m}^{2} \mathrm{~K}\right)$ \\
\hline$Q$ & Heat flux & $\mathrm{W} / \mathrm{m}^{2}$ \\
\hline$R_{s e}$ & External surface resistance & $\left(\mathrm{m}^{2} \mathrm{~K}\right) / \mathrm{W}$ \\
\hline$R_{s i}$ & Internal surface resistance & $\left(\mathrm{m}^{2} \mathrm{~K}\right) / \mathrm{W}$ \\
\hline$R_{H F}$ & Experimental thermal resistance of the system, obtained with heat flow-meter & $\left(\mathrm{m}^{2} \mathrm{~K}\right) / \mathrm{W}$ \\
\hline$R_{T}$ & Theoretical thermal resistance of the system & $\left(\mathrm{m}^{2} \mathrm{~K}\right) / \mathrm{W}$ \\
\hline$U$ & Thermal transmittance & $\mathrm{W} /\left(\mathrm{m}^{2} \mathrm{~K}\right)$ \\
\hline$U_{l a b}$ & Experimental thermal transmittance & $\mathrm{W} /\left(\mathrm{m}^{2} \mathrm{~K}\right)$ \\
\hline$U_{t}$ & Theoretical thermal transmittance & $\mathrm{W} /\left(\mathrm{m}^{2} \mathrm{~K}\right)$ \\
\hline$\varepsilon$ & Hemispherical emissivity of the surface & - \\
\hline$\theta_{e}$ & External air temperature & $\mathrm{K}$ \\
\hline$\theta_{i}$ & Internal air temperature & $\mathrm{K}$ \\
\hline$\theta_{s e}$ & External surface temperature & $\mathrm{K}$ \\
\hline$\theta_{s i}$ & Internal surface temperature & $\mathrm{K}$ \\
\hline$\theta_{\text {REF }}$ & Reflected temperature & $\mathrm{K}$ \\
\hline$\theta_{\text {mean }}$ & Mean between temperature of the surface and reflected temperature & $\mathrm{K}$ \\
\hline$\theta_{m}$ & $\begin{array}{l}\text { Mean between thermodynamic temperature of the surface and thermodynamic } \\
\text { temperature of its surroundings }\end{array}$ & $\mathrm{K}$ \\
\hline$v_{s e}$ & Wind speed adjacent to the external surface & $\mathrm{m} / \mathrm{s}$ \\
\hline$v_{s i}$ & Wind speed adjacent to the internal surface & $\mathrm{m} / \mathrm{s}$ \\
\hline$v_{s e(X P S)}$ & Wind speed adjacent to the external surface of XPS layer & $\mathrm{m} / \mathrm{s}$ \\
\hline$\sigma$ & Stefan-Boltzmann constant $\left(\sigma=5.6704 \times 10^{-8}\right)$ & $\mathrm{W} /\left(\mathrm{m}^{2} \mathrm{~K}^{4}\right)$ \\
\hline
\end{tabular}




\subsection{Theoretical method}

Consider a multilayer system composed by a set of plane and thermally homogeneous layers of infinite extent (in the $x$ and $z$ directions), as shown in figure 1 . This system is surrounded by air - Medium 0 and Medium $\mathrm{m}+1$. Assuming that Medium 0 (in contact with the inner surface) has a constant and higher temperature $\left(\theta_{i}\right)$ than Medium $\mathrm{m}+1$ (in contact with the outer surface and with a constant temperature of $\theta_{e}$ ), the heat flows from Medium 0 to Medium $m+1$. Based on the assumptions that we are in the presence of a steady-state heat transfer process and that there is energy conservation, the heat flow between the outer surface and the environment is equal to the heat flow between environments. Thus, the heat flow, $Q$, between internal and external environments, given by Eq. (1), is equal to the heat flow between inside and outside the system, given by Eq. (2).

$$
\begin{gathered}
Q_{x}=U \times\left(\theta_{i}-\theta_{e}\right) \\
Q_{x}=\frac{1}{R_{s e}} \times\left(\theta_{s e}-\theta_{e}\right) .
\end{gathered}
$$

Eq. (2) translates heat flow due to convection and radiation phenomena, where $R_{s e}$ is the thermal resistance, involving both phenomena, on the outer surface surrounding. The heat transfer due to thermal radiation is given by the Stefan-Boltzmann law, while convection is governed by Newton's law. Convection depends on wind speed $(v)$ near the surface [34] and radiation is influenced by the reflected temperature and the hemispherical emissivity of the surface [5].

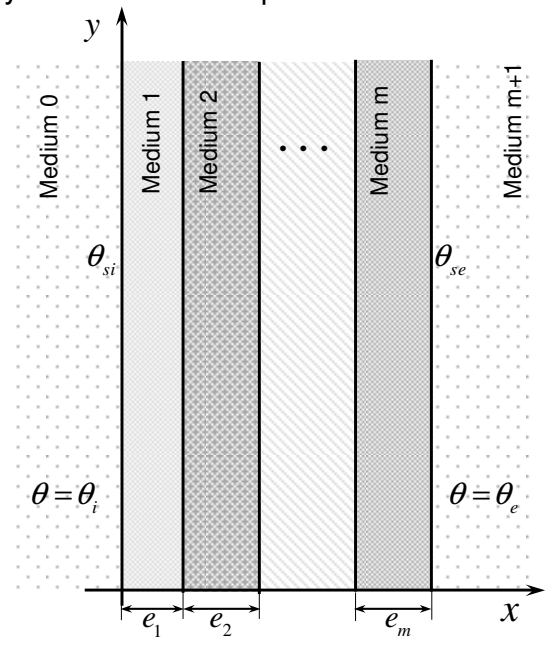

Fig. 1. Geometry of the problem.

The assumption described above was used by different authors ([16], [17] and [18]) that have proposed the following expressions to evaluate the U-value.

- $\quad$ Proposed by Albaciti and Tonelli [16]

$$
U=\frac{\varepsilon \times \sigma \times\left(\theta_{s e}^{4}-\theta_{e}^{4}\right)+h_{c}\left(\theta_{s e}-\theta_{e}\right)}{\left(\theta_{i}-\theta_{e}\right)}
$$

- $\quad$ Adapted from Fokaides and Kalogirou [17]

$$
U=\frac{4 \times \varepsilon \times \sigma \times \theta_{s e}{ }^{3} \times\left(\theta_{s e}-\theta_{R E F}\right)+h_{c}\left(\theta_{s e}-\theta_{e}\right)}{\left(\theta_{i}-\theta_{e}\right)}
$$

- $\quad$ Adapted from Vollmer and Möllmann [18]

$$
U=\frac{4 \times \mathcal{E} \times \sigma \times \theta_{\text {mean }}^{3}\left(\theta_{\text {se }}-\theta_{R E F}\right)+h_{c}\left(\theta_{\text {se }}-\theta_{e}\right)}{\left(\theta_{i}-\theta_{e}\right)}, \text { with } \quad \theta_{\text {mean }}=\frac{\left(\theta_{\text {se }}+\theta_{R E F}\right)}{2}
$$


In these equations the convective coefficient $\left(h_{c}\right)$, which depends on the wind speed acting on the surface, is given by $3.8054 \times v$, where $v$ is the wind speed near the surface. Typical values for the coefficient of convection, for inside walls are of the order of 2 to $8 \mathrm{~W} /\left(\mathrm{m}^{2} \mathrm{~K}\right)$ [18]. In these works, the use of infrared thermography (IRT) technique is proposed deal with the radiation phenomenon. Using the IRT camera, the radiation released from the surfaces is measured and converted into surface temperatures. The IRT can be used to measures the surface temperatures, air temperatures (using a paperboard as suggested by [18]), reflected temperature and emissivity. However, in the present paper the air temperatures and internal surface temperature were measure with thermocouples.

\section{Experimental procedure}

To evaluate the thermal transmittance using IRT, and following the methods proposed by Albaciti and Tonelli [16], P Fokaides and Kalogirou [17] and Vollmer and Möllmann [18], a $0.5 \times 0.5 \times 0.5 \mathrm{~m}^{3}$ box was built using different materials (figure 2). Each lateral sides of the box were built with four materials (approximately $20 \mathrm{~mm}$ thick): Medium-Density Fiberboard (MDF); Extruded Polystyrene (XPS); Natural Cork (NC); Cement Bonded Particle Board (CBPB). The top and the bottom of the box are closed off by an XPS board with $80 \mathrm{~mm}$ thickness to ensure there is very low heat flux going through the top and bottom. Only the lateral sides were studied.

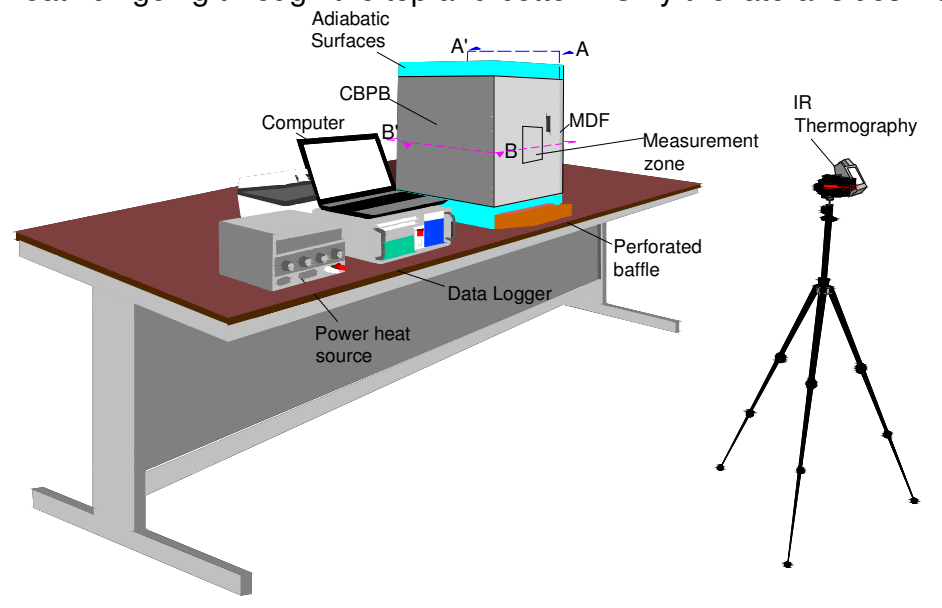

a)

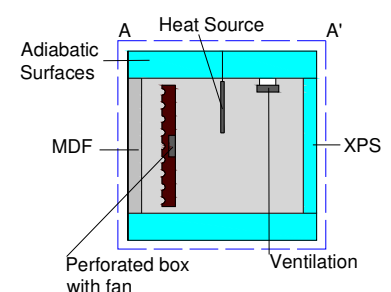

with fan

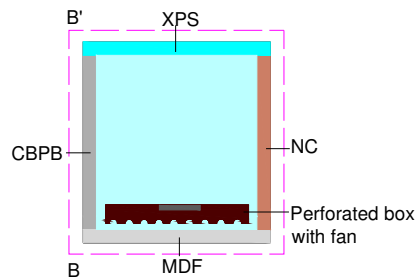

b)

Fig. 2. Test scheme: a) global scheme composed by the box, infrared thermography camera, computer, data logger, heat power source; b) scheme of the plant and cross section of the box composed by the perforated box, fans and heat source.

Tests measuring the temperature of external surface were performed using a Flir infrared camera, model T360. This was also the equipment used to estimate the surface emissivity and the reflected temperature. The relative humidity of the air was obtained by means of a datalogger Hygrolog HL-NT2-DP from Rotronic.

In order to apply Eqs. (3), (4) and (5), other parameters were also measured, such as the air speed inside and outside the box and the air and superficial temperature inside the box. These temperatures were measured using type $T$ (copper) thermocouples made of $0.2 \mathrm{~mm}$ wire, which were calibrated beforehand by an accredited laboratory. The data were recorded using a Yokogawa MW 100 data logger, with a time interval of $10 \mathrm{~s}$. The wind speed was measured with an anemometer model 4500, from Kestrel.

Heat flux meters were used in addition to the IRT technique. This is a contact method and should only be applied to elements with surfaces without irregularities. The equipment used to record the temperatures and heat fluxes on the test sample inner surfaces is a transverse gradient heat flux sensor (TRSYS01 from Hukseflux), also known as a heat flow-meter. This equipment is composed of two HFP01 heat flux sensors, four thermocouples and a data logger to record measurements. The heat flux sensors were applied to the surfaces inside and outside of the box, for each tested material. Two thermocouples were also installed close to the heat flux sensors to register surface temperatures. By knowing the heat flow through the solution it is possible to calculate the thermal resistance [22].

\subsection{Measurements procedure}

The experiments were performed while imposing a steady heat flow rate from the inside to the outside of the box. Steady state conditions were ensured by controlling the power of the heat source (placed inside the box) and a set of fans (two implemented inside and three positioned outside the box). The set of fans and a perforated baffle (box) (Fig. 2b)) were used to provide uniform heating and control the convection phenomenon. The tests were carried out in a 
controlled environment laboratory with a set-point temperature of $(23 \pm 2){ }^{\circ} \mathrm{C}$ and $(50 \pm 5) \%$ relative humidity, allowing the creation of a thermal gradient between the inside and the outside of the box.

The conversion of infrared radiation into temperature depends on the distance between the camera and the surface, the relative humidity, air temperature, the emissivity and the reflected temperature. However, the exterior surface temperature $\left(\theta_{s e}\right)$ measured with the infrared camera which is introduced in the equations mentioned does not take into account the reflected temperature.

The emissivity was obtained according the empirical procedure, provided in ASTM E 1933 - 99 [27], using a black adhesive tape with known emissivity. This procedure consists on changing the emissivity of the measured surface in order to match the temperature of the black tape. The reflective temperature was estimated according with the ASTM 1862-97 [28], using a crumpled up aluminium paper and measuring its mean temperature.

The measurements used to estimate the thermal transmittance were made when the steady state was reached. At this time all parameters (emissivity, reflected temperature, distance between object and infrared camera, air relative humidity and air temperature) were registered. The temperatures measured by thermocouples were recorded for at least one hour. Along this period, thermograms were record every 10 minutes, to ensure that the thermal equilibrium of the system was maintained. The parameters used in the calculations were obtained using the mean temperature recorded by the thermocouples and the mean temperature registered in the thermograms (considering only the central area of the test specimens).

The heat flow-meter, used to record the heat fluxes and temperatures of each tested material, was placed away from the boundaries of the specimen in order to avoid the influence of the edges of the box on the results (its position is indicated in the thermograms shown in figure 3). For each layer of material in the box, one HFP01 heat flux sensors and two thermocouples were attached to its outside surface, while another sensor and two other thermocouples were attached to its inside surface. The inner and outer sensors were applied on opposite sides at similar coordinates, but without interfering with the infrared measurements. The thermal resistance of a particular layer is given by the ratio between the heat flow that goes through the layer and the temperature difference between its inner and outer surfaces.

\section{Results}

In order to evaluate the $U$-value results obtained using the infrared thermography, the materials were previously characterized. Thermal conductivity was found by the Guarded Hot-Plate Method (ISO 8302:1991 [21]) using a LambdaMebtechnik GmbH Dresden apparatus, a single-specimen Lambda-meter EP-500 model, following the test procedure defined in EN 12667:2001 [33]. The thermal conductivity and thickness of each material are presented in table 2.

Table 2. Thickness and thermal conductivity of each material

\begin{tabular}{|l|l|l|}
\hline Material & Thickness $(\mathrm{mm})$ & Thermal conductivity \\
\hline XPS & 20.00 & 0.039 \\
\hline MDF & 18.17 & 0.120 \\
\hline Natural cork & 20.60 & 0.047 \\
\hline CBPB & 18.33 & 0.220 \\
\hline
\end{tabular}

The wind speed measured in the vicinity of the layer surfaces was constant throughout all laboratorial tests. The value obtained on inner surface was $v_{s i}=0.3 \mathrm{~m} / \mathrm{s}$, while the wind speed measured in the vicinity of the outer surface was $v_{s e}=0.4 \mathrm{~m} / \mathrm{s}$. The wind speed in the vicinity of the outer surface XPS was $v_{s e(X P S)}=0.0 \mathrm{~m} / \mathrm{s}$ in order to guarantee a higher thermal gradient.

Several tests were performed to ensure the reproducibility of results. For this reason, at least three laboratorial tests were made for each material. The parameters measured in each experiment are presented in table 3 , namely, the external and internal surface temperature, external and internal air temperature, reflected temperature and emissivity. As an example of the external surface temperature measurements made with IRT, the $1^{\text {st }}$ and $3^{\text {rd }}$ test for MDF are presented in figure 3 . The temperature used in the calculations is the average temperature recorded in the square represented in this figure. It was verified that the temperature distribution on that area was always homogeneous.

\subsection{Theoretical thermal transmittance}

The results obtained, according the proposed method, were compared with the theoretical thermal transmittance $-U_{t}$. This coefficient was determined according to Eq. (6), following the ISO 6946:2007 [5]:

$$
U_{t}=\frac{1}{R_{s i}+\sum_{j} \frac{e_{j}}{\lambda_{j}}+R_{s e}}
$$


where $R_{s i}=\frac{1}{h_{c}+h_{r}} ; h_{c_{i}}=3.8054 \times v_{i} ; \quad h_{r_{i}}=4 \times \varepsilon \times \sigma \times \theta_{m}^{3}$, with $\theta_{m}=\frac{\theta_{s i}+\theta_{i}}{2} \quad$ and $R_{s e}=\frac{1}{h_{c_{e}}+h_{r_{e}}} ; \quad h_{c_{e}}=3.8054 \times v_{e} ; \quad h_{r_{e}}=4 \times \varepsilon \times \sigma \times \theta_{m}^{3}$, with $\theta_{m}=\frac{\theta_{s e}+\theta_{e}}{2}$

Table 3. Parameters obtained in each test

\begin{tabular}{|c|c|c|c|c|c|c|c|}
\hline Material & & $\theta_{s e}(\mathrm{~K})$ & $\theta_{s i}(\mathrm{~K})$ & $\theta_{e}(\mathrm{~K})$ & $\theta_{i}(\mathrm{~K})$ & $\theta_{R E F}(\mathrm{~K})$ & Emissivity $(\varepsilon)$ \\
\hline \multirow{3}{*}{ XPS } & $1^{\mathrm{st}}$ test & 300.95 & 308.69 & 297.51 & 310.65 & 298.05 & \multirow{3}{*}{0.96} \\
\hline & $2^{\text {nd }}$ test & 298.85 & 306.29 & 295.34 & 307.69 & 296.45 & \\
\hline & $3^{\text {rd }}$ test & 303.25 & 310.25 & 299.80 & 312.42 & 300.45 & \\
\hline \multirow{3}{*}{ MDF } & $1^{\text {st }}$ test & 297.45 & 303.25 & 293.25 & 305.12 & 293.05 & \multirow{3}{*}{0.94} \\
\hline & $2^{\text {nd }}$ test & 298.55 & 303.25 & 294.75 & 305.40 & 295.55 & \\
\hline & $3^{\text {rd }}$ test & 298.55 & 303.59 & 294.35 & 305.74 & 295.35 & \\
\hline \multirow{3}{*}{ Natural Cork } & $1^{\text {st }}$ test & 300.15 & 308.16 & 297.01 & 309.54 & 298.05 & \multirow{3}{*}{0.95} \\
\hline & $2^{\text {nd }}$ test & 299.45 & 307.82 & 296.23 & 308.98 & 297.05 & \\
\hline & $3^{\text {rd }}$ test & 298.75 & 307.55 & 295.43 & 307.88 & 296.45 & \\
\hline \multirow{3}{*}{ CBPB } & $1^{\text {st }}$ test & 301.55 & 305.92 & 296.73 & 308.76 & 297.15 & \multirow{3}{*}{0.96} \\
\hline & $2^{\text {nd }}$ test & 301.75 & 304.38 & 295.55 & 307.99 & 296.85 & \\
\hline & $3^{\text {rd }}$ test & 299.95 & 302.87 & 294.88 & 306.63 & 296.15 & \\
\hline
\end{tabular}

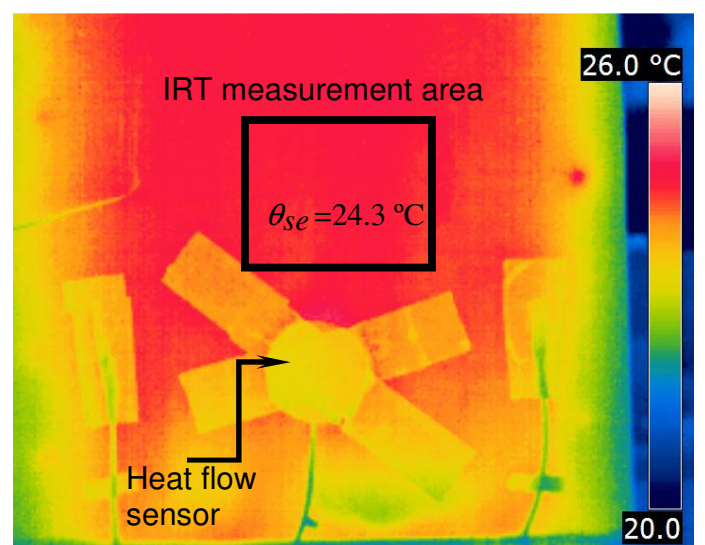

a)

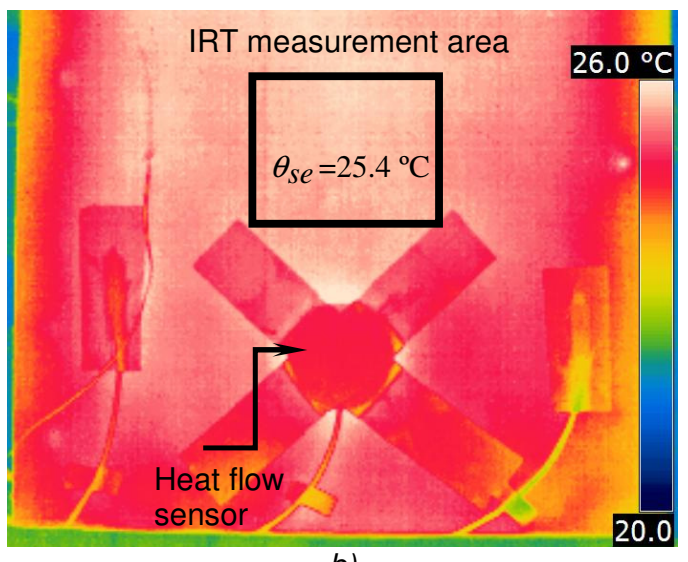

b)

Fig. 3. Thermograms of external surface temperature of MDF: a) $1^{\text {st }}$ test; b) $2^{\text {nd }}$ test

\subsection{Presentation and discussion of experimental results}

This section presents the experimental thermal transmittance results obtained by applying Eqs. (3) to (5) for each material (XPS, MDF, NC and CBPB). In the following paragraphs these results are compared with the experimental thermal transmittance calculated according to Eq. (6). and the thermal resistances evaluated with infrared thermography are also compared with those obtained from heat flux sensor measurements, together with the surface temperatures.

The following parameter $(E)$ was computed to evaluate the deviation between the experimental and theoretical heat transmittance:

$$
E=100 \times \frac{\left|U_{t}-U_{l a b}\right|}{U_{t}}
$$

Table 4 lists the experimental thermal transmittance results obtained theoretical and experimentally. The relative error ( $E$ ) for each approach given by Eqs. (3), (4) and (5) is presented and the maximum relative error $\left(E_{\max }\right)$ for each test is also given. The worst result presents a relative error around $37 \%$ (obtained for the Eq. (3) in the case of insulating materials). For the other two equations the errors are lower than 13.64\%. The thermal insulations materials are the ones that presented the worst results. The tests performed using CBPB and MDF (materials with less thermal resistance) list errors below $30 \%$. The best results were obtained using Eq. (4), resulting in errors between $1.28 \%$ and $13.64 \%$.

Table 4. Theoretical and experimental thermal transmittance results for each material 


\begin{tabular}{|c|c|c|c|c|c|c|c|c|c|}
\hline \multirow{2}{*}{ Material } & \multirow{2}{*}{ Test } & \multirow{2}{*}{$\begin{array}{c}U_{t} \\
\left(\mathrm{~W} / \mathrm{m}^{2} \cdot \mathrm{K}\right)\end{array}$} & \multicolumn{3}{|c|}{$U_{L a b} \mathrm{~W} /\left(\mathrm{m}^{2} \mathrm{~K}\right)$} & \multicolumn{3}{|c|}{$E(\%)$} & \multirow{2}{*}{$E_{\max }(\%)$} \\
\hline & & & Eq. (3) & Eq. (4) & Eq. (5) & Eq. (3) & Eq. (4) & Eq. (5) & \\
\hline \multirow{3}{*}{ XPS } & $1^{\text {st }}$ test & 1.23 & 1.53 & 1.31 & 1.29 & 24.30 & 6.60 & 5.07 & 24.30 \\
\hline & $2^{\text {nd }}$ test & 1.22 & 1.62 & 1.13 & 1.12 & 33.16 & 7.33 & 8.44 & 33.16 \\
\hline & $3^{\text {rd }}$ test & 1.24 & 1.63 & 1.35 & 1.33 & 31.88 & 8.88 & 7.38 & 31.88 \\
\hline \multirow{3}{*}{ MDF } & $1^{\text {st }}$ test & 2.30 & 2.48 & 2.62 & 2.57 & 7.73 & 13.64 & 11.65 & 13.64 \\
\hline & $2^{\text {nd }}$ test & 2.31 & 2.53 & 2.14 & 2.12 & 9.35 & 7.42 & 8.46 & 9.35 \\
\hline & $3^{\text {rd }}$ test & 2.31 & 2.61 & 2.16 & 2.13 & 12.78 & 6.86 & 7.96 & 12.78 \\
\hline \multirow{3}{*}{$\begin{array}{l}\text { Natural } \\
\text { Cork }\end{array}$} & $1^{\text {st }}$ test & 1.41 & 1.82 & 1.36 & 1.35 & 29.06 & 3.64 & 4.36 & 29.06 \\
\hline & $2^{\text {nd }}$ test & 1.41 & 1.82 & 1.47 & 1.46 & 29.54 & 4.75 & 3.83 & 29.54 \\
\hline & $3^{\text {rd }}$ test & 1.40 & 1.91 & 1.47 & 1.46 & 36.28 & 4.55 & 3.68 & 36.28 \\
\hline \multirow{3}{*}{ CBPB } & $1^{\text {st }}$ test & 2.83 & 2.95 & 2.79 & 2.75 & 4.08 & 1.28 & 2.96 & 4.08 \\
\hline & $2^{\text {nd }}$ test & 2.82 & 3.65 & 3.12 & 3.06 & 29.63 & 10.64 & 8.62 & 29.63 \\
\hline & $3^{\text {rd }}$ test & 2.79 & 3.13 & 2.56 & 2.52 & 12.05 & 8.42 & 9.70 & 12.05 \\
\hline
\end{tabular}

Table 5 presents the thermal resistance obtained for each material (without considering the thermal surface resistance) using the heat flow-meter measurements - $R_{H F}$, considering the $1^{\text {st }}$ test results. These results are compared with the theoretical thermal resistance - $R_{T}$ - and also with the results given by the IRT measurements $R_{\text {Lab }}$. The maximum relative error between theoretical and IRT thermal resistance results $\left(R_{T}\right.$ vs $\left.R_{\text {Lab }}\right)$ and between theoretical and heat flow-meter results $\left(R_{T}\right.$ vs $\left.R_{H F}\right)$ are given. The heat flow-meter errors are greater than those obtained from IRT application, except in the case of XPS.

Table 5. Thermal resistance of each material: theoretical and experimental results with IRT and heat flow-meter measurements.

\begin{tabular}{|l|l|c|c|c|c|c|c|}
\hline \multirow{2}{*}{ Material } & \multirow{2}{*}{$R_{T}\left(\mathrm{~m}^{2} \mathrm{~K}\right) / \mathrm{W}$} & \multicolumn{3}{|c|}{$R_{\text {Lab }}\left(\mathrm{m}^{2} \mathrm{~K}\right) / \mathrm{W}$} & \multirow{2}{*}{$R_{H F}\left(\mathrm{~m}^{2} \mathrm{~K}\right) / \mathrm{W}$} & \multicolumn{2}{c|}{$E_{\max }(\%)$} \\
\cline { 3 - 5 } \cline { 8 - 9 } & & Eq. (3) & Eq. (4) & Eq. (5) & & $R_{\text {Lab }}$ vs $R_{T}$ & $R_{H F}$ vs $R_{T}$ \\
\hline XPS & 0.5110 & 0.3519 & 0.4606 & 0.4717 & 0.4662 & 24.30 & 8.77 \\
\hline MDF & 0.1514 & 0.1203 & 0.0993 & 0.1061 & 0.2200 & 13.64 & 45.29 \\
\hline Natural Cork & 0.4383 & 0.2785 & 0.4651 & 0.4707 & 0.5762 & 29.06 & 31.47 \\
\hline CBPB & 0.0833 & 0.0695 & 0.0879 & 0.0941 & 0.1171 & 4.08 & 40.52 \\
\hline
\end{tabular}

\subsection{Sensitivity analysis}

To better understand the influence of each parameter on final results, a sensitivity analysis was performed. This analysis considered variations regarding emissivity, wind speed and reflected temperature for MDF measurements. Note that the emissivity measurement affects the surface temperature registered by the infrared camera, which is incorporated in the following results.

Tables 6, 7 and 8 list the results obtained when the emissivity, wind speed and reflected temperature for MDF measurements are changed. The real measurements are also listed for comparison and are identified by the cells filled in in grey colour. In these tables the relative error is also given, for better understanding of the influence of the parameters.

The results given in table 6 show relative errors lower than $33 \%$. The relative errors obtained with $\varepsilon=0.89$ are greater than when assuming higher emissivity.

Table 6. Relative error (\%) assuming the variation of the MDF emissivity value.

\begin{tabular}{|c|c|c|c|c|c|c|c|c|c|c|}
\hline \multirow{2}{*}{$\begin{array}{c}U_{t} \\
\mathrm{~W} /\left(\mathrm{m}^{2} \mathrm{~K}\right)\end{array}$} & \multirow{2}{*}{ Eq. } & \multicolumn{3}{|c|}{$\varepsilon=0.89$} & \multicolumn{3}{|c|}{$\varepsilon=0.94$} & \multicolumn{3}{|c|}{$\mathcal{E}=0.99$} \\
\hline & & $\theta_{s e}(\mathrm{~K})$ & $\begin{array}{c}U_{L a b} \\
\mathrm{~W} /\left(\mathrm{m}^{2} \mathrm{~K}\right)\end{array}$ & $E(\%)$ & $\theta_{s e}(\mathrm{~K})$ & $\begin{array}{c}U_{L a b} \\
\mathrm{~W} /\left(\mathrm{m}^{2} \mathrm{~K}\right)\end{array}$ & $E(\%)$ & $\theta_{s e}(\mathrm{~K})$ & $\begin{array}{c}U_{L a b} \\
\mathrm{~W} /\left(\mathrm{m}^{2} \mathrm{~K}\right)\end{array}$ & $E(\%)$ \\
\hline \multirow{3}{*}{2.30} & (3) & \multirow{3}{*}{298.65} & 3.07 & 33.42 & \multirow{3}{*}{297.45} & 2.48 & 7.77 & \multirow{3}{*}{296.35} & 1.90 & 17.51 \\
\hline & (4) & & 3.23 & 40.20 & & 2.62 & 13.68 & & 2.02 & 12.20 \\
\hline & (5) & & 3.16 & 37.13 & & 2.57 & 11.69 & & 2.00 & 13.38 \\
\hline
\end{tabular}


Wind speed influences the convection coefficient. For this reason, table 7 lists the relative errors obtained considering different values of wind speed in the vicinity of the outer surface of the MDF layer. It can be seen that experimental thermal transmittance results decrease for lower wind speed values.

Table 7. Relative error (\%) assuming the variation of wind speed variance in the outer surface of the MDF layer.

\begin{tabular}{|c|c|c|c|c|c|c|c|c|c|}
\hline \multirow{2}{*}{$\begin{array}{c}U_{t} \\
\left(\mathrm{~W} / \mathrm{m}^{2} . \mathrm{K}\right)\end{array}$} & \multirow{2}{*}{\begin{tabular}{c} 
Eq. \\
\cline { 2 - 11 }
\end{tabular}} & $\begin{array}{c}U_{\text {Lab }} \\
\mathrm{W} /\left(\mathrm{m}^{2} \mathrm{~K}\right)\end{array}$ & $E(\%)$ & $\begin{array}{c}U_{\text {Lab }} \\
\mathrm{W} /\left(\mathrm{m}^{2} \mathrm{~K}\right)\end{array}$ & $E(\%)$ & $\begin{array}{c}U_{\text {Lab }} \\
\mathrm{W} /\left(\mathrm{m}^{2} \mathrm{~K}\right)\end{array}$ & $E(\%)$ & $\begin{array}{c}U_{\text {Lab }} \\
\mathrm{W} /\left(\mathrm{m}^{2} \mathrm{~K}\right)\end{array}$ & $E(\%)$ \\
\hline \multirow{3}{*}{2.30} & $(3)$ & 1.94 & 15.60 & 2.21 & 3.96 & 2.48 & 7.77 & 2.75 & 19.41 \\
\cline { 2 - 12 } & $(4)$ & 2.08 & 9.74 & 2.35 & 1.95 & 2.62 & 13.68 & 2.89 & 25.32 \\
\cline { 2 - 12 } & $(5)$ & 2.03 & 11.73 & 2.30 & 0.04 & 2.57 & 11.69 & 2.84 & 23.33 \\
\hline
\end{tabular}

Reflected temperature has effects on the results obtained by the proposed equations. Table 8 shows the relative error obtained when the reflected temperature changes $\pm 1^{\circ} \mathrm{C}$. Table 8 indicates, as expected, that results from Eq. (3) don't change when other values of reflected temperature are assumed, because the authors did not consider this parameter. The errors obtained with Eqs. (4) and (5) increase for lower reflected temperatures.

Table 8. Relative error (\%) for a reflected temperature variance of $1^{\circ} \mathrm{C}-M D F$ layer.

\begin{tabular}{|c|c|l|l|l|l|l|c|}
\hline \multirow{2}{*}{$\begin{array}{c}U_{t} \\
\left(\mathrm{~W} / \mathrm{m}^{2} . \mathrm{K}\right)\end{array}$} & \multirow{2}{*}{ Eq. } & \multicolumn{2}{|c|}{$\theta_{\text {REF }}=292.05 \mathrm{~K}$} & \multicolumn{2}{c|}{$\theta_{\text {REF }}=293.05 \mathrm{~K}$} & \multicolumn{2}{c|}{$\theta_{\text {REF }}=294.05 \mathrm{~K}$} \\
\cline { 3 - 8 } & $\begin{array}{c}U_{\text {Lab }} \\
\mathrm{W} /\left(\mathrm{m}^{2} \mathrm{~K}\right)\end{array}$ & $E(\%)$ & $\begin{array}{c}U_{\text {Lab }} \\
\mathrm{W} /\left(\mathrm{m}^{2} \mathrm{~K}\right)\end{array}$ & $E(\%)$ & $\begin{array}{c}U_{\text {Lab }} \\
\mathrm{W} /\left(\mathrm{m}^{2} \mathrm{~K}\right)\end{array}$ & $E(\%)$ \\
\hline \multirow{3}{*}{2.30} & $(3)$ & 2.48 & 7.77 & 2.48 & 7.77 & 2.48 & 7.77 \\
\cline { 2 - 8 } & $(4)$ & 3.09 & 34.20 & 2.62 & 13.68 & 2.15 & 6.84 \\
\cline { 2 - 8 } & $(5)$ & 3.02 & 31.21 & 2.57 & 11.69 & 2.12 & 8.03 \\
\hline
\end{tabular}

\section{Conclusions}

The present paper describes an experimental procedure to evaluate the thermal transmittance results obtained by applying three distinct methods which have been proposed by authors ([16], [17] and [18]), in which infrared thermography is used to measure surface temperature. This evaluation was performed in a laboratorial space under controlled conditions. A box was built and monitored allowing for the study of four materials (MDF, XPS, NC and CBPB). A sensitivity analysis was also performed in order to assess the influence of the parameters involved in the calculations.

The smaller relative errors of thermal transmittance obtained were given by using the method proposed by P. A. Fokaides and S. A. Kalogirou [17] which showed errors between $1.28 \%$ and $13.64 \%$.

A sensitivity analysis demonstrated that the thermal transmittance values decrease when lower emissivity, wind speed or reflected temperature values are assumed.

\section{Acknowledgments}

The research work presented herein was supported by the Portuguese Foundation for Science and Technology (FCT), under research project PTDC/ECM/114189/2009. This work has also been supported by the Energy and Mobility for Sustainable Regions - EMSURE - Project (CENTRO-07-0224-FEDER-002004).

\section{REFERENCES}

[1] European Directive 2010/31/UE of the European Parliament and of the Council of 19 May 2010 on the energy performance of buildings (recast). Official Journal of the European Union L 153, pp. 0013 - 0035, Strasbourg, 2010.

[2] European Directive 2002/91/EC of the European Parliament and of the Council of 116 December 2002 on the energy performance of buildings. Official Journal of the European Union L 1, pp. 0065 - 0070, Brussels, 2003.

[3] International Organization for Standardization, ISO 13789: Thermal performance of buildings - Transmission and ventilation heat transfer coefficients - Calculation method, 2007.

[4] International Organization for Standardization, ISO 13790:2008 - Energy performance of buildings - Calculation of energy use for space heating and cooling, 2008.

[5] International Organization for Standardization, ISO 6946:2007 - Building components and building elements Thermal resistance and thermal transmittance - Calculation method, 2007.

[6] International Organization for Standardization, ISO 10211: Thermal bridges in building construction - Heat flows and surface temperatures - Detailed calculations, 2007.

[7] International Organization for Standardization, ISO 8990: Thermal insulation - Determination of steady state thermal transmission properties - Calibrated and guarded hot box, 1996. 
[8] American Society for Testing and Materials ASTM C1363-05: Standard Test Method for Thermal Performance of Building Materials and Envelope Assemblies by Means of a Hot Box Apparatus, West Conshohocken, PA, 2005.

[9] Interstate Standard of Russian Federation GOST 26602.1-99 - Windows and doors. Methods of determination of resistance of thermal transmission, 1999.

[10] F. Asdrubali, and G. Baldinelli, "Thermal transmittance measurements with the hot box method: Calibration, experimental procedures, and uncertainty analyses of three different approaches", Energy and Buildings, vol. 43 (7), pp. 1618-1626, 2011.

[11] Y. Gao, J. J. Roux, C. Teodosiu, and L.H. Zhao, "Reduced linear state model of hollow blocks walls, validation using hot box measurements", Energy and Buildings, vol. 36, pp. 1107-1115, 2004.

[12] N. Simões, I. Simões, A. Tadeu, C.A.B. Vasconcellos, and W.J. Mansur, "3D transient heat conduction in multilayer systems and experimental validation of semi-analytical solution", International Journal of Thermal Sciences, vol. 57, pp. 192-203, 2012.

[13] D.M. Burch, R.R. Zarr, and B.A. Licitra, "Dynamic test method for determining transfer function coefficients for a wall specimen using a calibrated hot box, insulation materials", American Society for Testing and Materials, ASTM STP 1030, Testing and Applications, West Conshohocken, PA, pp. 345-361, 1990.

[14] L. F. Cabeza, A. Castell, M. Medrano, I. Martorell, G. Pérez, I. Fernández, "Experimental study on the performance of insulation materials in Mediterranean construction”, Energy and Buildings 42 (5), pp. 630 - 636 , 2010.

[15] R. R. Zarr, "A history of testing heat insulators at the national institute of standards and technology", ASHRAE Transactions 107, pp. $661-671,2001$.

[16] R. Albaciti and A. M. Tonelli, "Infrared thermovision technique for the assessment of thermal transmittance value opaque building elements on site", Energy and Buildings 42, pp. 2177-2183, 2010.

[17] P. A. Fokaides and S. A. Kalogirou, "Application of infrared thermography for the determination of the overall heat transfer coefficient (U - Value) in building envelopes", Applied Energy 88, pp. 4358-4365, 2011.

[18] M. Vollmer and K. P. Möllmann, Infrared Thermal Imaging - Fundamentals, Research and Applications, pp. 391-394, 2010.

[19] G. Desogus, S. Mura, and R. Ricciu, "Comparing different approaches to in situ measurement of building components thermal resistance", Energy and Buildings vol. 43 (10), pp. 2613-2620, 2011.

[20] International Organization for Standardization, ISO 8301: Thermal insulation - Determination of steady-state thermal resistance and related properties - Heat flow meter apparatus, 1991.

[21] International Organization for Standardization, ISO 8302: Thermal insulation - Determination of steady-state thermal resistance and related properties - Guarded hot plate apparatus, 1991.

[22] International Organization for Standardization, ISO 9869: Thermal insulation - Building elements - In-situ measurement of thermal resistance and thermal transmittance, 1994.

[23] A. Tadeu, N. Simões, I. Simões, F. Pedro and L. Skerget, "In - situ thermal resistance of walls using an iterative dynamic model", Int. Journal for Housing Science, Vol. 38, No.1 pp.1 - 12, 2014.

[24] E. Barreira, V. P. Freitas, "Evaluation of building materials using infrared thermography", Construction and Building Materials 21, pp. 218-224, 2007.

[25] C.A. Balaras, A.A. Argiriou, "Infrared thermography for buildings diagnostics", Energy and Buildings 34 pp. 171183, 2002.

[26] N.P. Avdelidis, A. Moropoulou, "Emissivity considerations in building thermography", Energy and Buildings 35, pp. 663-667, 2003.

[27] American Society for Testing and Materials, ASTM Standard E1933 - 99, Standard Test Methods for Measuring and Compensating for Emissivity Using Infrared Imaging Radiometers, West Conshohocken, PA, pp. 1-3, 1999.

[28] American Society for Testing and Materials, ASTM Standard E1862-97, Standard Test Methods for Measuring and Compensating for Reflected Temperature Using Infrared Imaging Radiometers, West Conshohocken, PA, pp. 1-3, 1997.

[29] J. R. V. Monteiro, S. M. Leder, "A aplicação da Termografia como ferramenta de investigação térmica no espaço urbano", elecs, VITÓRIA2011, 2011.

[30] M. G. Emmel, M. O. Abadie, N. Mendes, "New external convective heat transfer coefficient correlations for isolated low-rise buildings", Energy and Buildings 39 pp. 335-342, 2007.

[31] Madding, R., "Finding R-Values of SIP and ICF Wall Construction with IR thermography", Inframation Proceedings Vol. 10, pp. 37-47, 2009.

[32] International Organization for Standardization, ISO 8302:1991. Thermal Insulation - Determination of steady state thermal resistance and related properties - Guarded Hot-Plate apparatus, 1991.

[33] European Standard, EN 12667:2001. Thermal performance of building materials and products. Determination of thermal resistance by means of guarded hot plate and heat flow meter methods. Products of high and medium thermal resistance, 2001.

[34] J. A. Palyvos, "A survey of wind convection coefficient correlations for building envelope energy systems' modeling", Applied Thermal Engineering, vol (28), pp. 801-808, (2008). 